\title{
Doxorubicin-loaded biocompatible hydroxyethyl cellulose hemostatic gauze in situ gel for controlled release of drugs and prevention of breast cancer recurrence post-surgery
}

Bai-Yi Yan ${ }^{1 \#}$, Bai-Lu Liu ${ }^{1 \#, ~ L i n g-Y u ~ Y i ", ~ G u i-L i n g ~ W a n g ~}{ }^{1}$, Bai-Zhong Xue ${ }^{2}$, Ying $\mathrm{Xie}^{1 *}$

${ }^{1}$ Beijing Key Laboratory of Molecular Pharmaceutics and New Drug Delivery Systems, Department of Pharmaceutics, School of Pharmaceutical Sciences, Peking University, Beijing 100191, P. R. China

2 Penglai Nuokang Pharmaceutical Co., Ltd. Shandong Province, Penglai, 265607, P. R. China

Number of pages: 6

Number of figures: 2

Number of tables: 1 


\section{Supporting Information}

\section{Standard of grading for Skin sensitization GB/T 21608-2008}

Table S1 Standard of grading for skin sensitization GB/T 21608-2008

\begin{tabular}{ccc}
\hline \multicolumn{3}{c}{ Standard of grading for Skin sensitization } \\
\hline erythematous response & score & Edema response \\
\hline Nothing & 0 & Nothing \\
Very slight (just visible) & 1 & Very slight (just visible) \\
Slight (pale red) & 2 & Slight (lower than 1mm) \\
Moderate (bright red, clear region) & 3 & Moderate (nearly 1mm) \\
Severe (purplish red, slight eschar) & 4 & Severe (higher than $1 \mathrm{~mm}$ ) \\
\hline
\end{tabular}

\section{Gel properties of GEL (DOX)}

The translucent glass tube containing 4mL GEL (DOX) solution was placed in a water bath at $25^{\circ} \mathrm{C}$ for 10 mins to reach heat balance and then gradually heated at a speed of $1{ }^{\circ} \mathrm{C} / \mathrm{min}$. The fluidity of gels in the tube was observed by frequently flipflopping. The momentary temperature at which the liquid stopped flowing was noted as the gelation temperature $T_{\text {sol-gel, }}$ a temperature at which the liquid phase converts into a gel state.

An empty glass vial with a magnetic bar $(10 \times 5 \mathrm{~mm})$ inside for stirring purpose was placed into a water bath at $37^{\circ} \mathrm{C}$. The incessant speed of the magnetic stirring was 500 rpm. $4.0 \mathrm{ml} \mathrm{GEL}(\mathrm{DOX})$ solution of $25^{\circ} \mathrm{C}$ was injected into the vial and the time at which the magnetic bar stopped rotation was recorded as the gelation time $t(\mathrm{~s})$.

\section{The release profile of GEL (DOX)}

For probing the velocity and degree of DOX release from GEL(DOX), the in vitro release behavior of DOX was monitored by using the dialysis-bag diffusion method described previously. ${ }^{1}$ A dialysis bag $(\mathrm{MWCO}=3500 \mathrm{Da})$ with $1 \mathrm{~mL}$ of prepared GEL(DOX) was placed in a conical flask with stopper, containing $30 \mathrm{ml}$ of $\mathrm{pH} 7.2 \mathrm{PBS}$ (phosphate-buffered saline, $0.01 \mathrm{~mol} / \mathrm{L}$ ), spun at $50 \mathrm{rpm}$ at $310 \pm 0.5 \mathrm{~K}$. At a predetermined time-point, $1 \mathrm{~mL}$ release medium was collected and replaced with $1 \mathrm{~mL}$ of fresh PBS. Free DOX solution $(15 \mathrm{mg} / \mathrm{mL})$ in water was used as the positive control, and $1 \mathrm{~cm} \times 1 \mathrm{~cm}$ HEC gauze dropped with GEL(DOX) was investigated how HEC affected the release of DOX. The amount of DOX released was evaluated using UV-vis 
spectrometer $(\lambda=484 \mathrm{~nm})$ (UV-1800PC, Shanghai Mapada Instrument Co., Ltd., Shanghai, China) and the release curve of cumulative drug release versus time was made accordingly. Cumulative drug release $(C R \%)$ was calculated with the following formula:

$$
\mathrm{CL} \%(\mathrm{n})=\frac{c_{n} \times 30+\sum_{i=1}^{n-1} c_{i} \times 1}{c_{f} \times 30+\sum_{i=1}^{n-1} c_{i} \times 1} \times 100 \%
$$

in which $c_{\mathrm{n}}$ is the concentration of DOX in the supernatant at a given time point, $c_{\mathrm{i}}$ is the concentration of DOX in the supernatant at a previously given time, $c_{\mathrm{f}}$ is the concentration of DOX in the supernatant when the dialysis bag opened at final, 30 is the volume of release medium, and 1 stands for the sample volume at each given time.

\section{Therapy efficacy of HEC-DOX for breast tumor}

\subsection{Methods}

Breast tumor-bearing BALB/c-nu nude mice were prepared by $200 \mu \mathrm{MCF}-7$ cells suspension at the concentration of $5 \times 10^{7}$ cells $/ \mathrm{mL}$ inoculated into the right armpit of the nude mice. After the tumor volume grew to about $100 \mathrm{~mm}^{3}$, the mice were anesthetized and then the tumor was removed to the remaining $20 \mathrm{~mm}^{3}$ under aseptic operation as tumor recurrence model.

Eighteen BALB/c-nu nude mice (female, weight of $20 \mathrm{~g}$ ) were randomly divided into 3 groups (6 mice in each group), including HEC-DOX, HEC, and blank control group. $0.03 \mathrm{~mL}$ DOX-HCl solution $(15 \mathrm{mg} \mathrm{DOX} / \mathrm{mL})$ was dropped on $1 \mathrm{~cm} \times 1 \mathrm{~cm} \mathrm{HEC}$ gauze and then covered to the residual tumor, the wound was closed by leaving HEC gauze in the body. HEC gauze without drug served as negative control group and the wound sutured directly after hemostasis with ordinary gauze was served as blank group. The survival state of animals was monitored and tumor size was measured every day for 14 days. One mouse in each group was sacrificed on the $4^{\text {th }}$ day after operation and tumor and major organs were separated for TUNEL staining, H\&E staining and blood routine test, respectively. 


\subsection{Results}

As the tumor was resected, HEC gauze was left on the operation site to stop bleeding, while the gelatinous microenvironment formed by its dissolution in tissue fluid provided adhesion for cell proliferation. Compared with blank control group, tumor in HEC group grew quickly as shown in Fig S1. However, DOX-HCl dropped onto HEC gauze inhibited tumor growth significantly in the first 6 days after operation (Fig S1A, B) that demonstrated the DOX was beneficial to therapy of tumor. During the treatment, there was not difference of body weight among these three groups (Fig S1C), but the mid-survival time of HEC-DOX was prolonged (Fig S1-D).

On the $4^{\text {th }}$ day after HEC-DOX treatment, the severely broken DNA strand shown in TUNEL staining indicated that DOX induced cell apoptosis (Fig S1-E). Blood routine test (Fig. S2-A) showed that the white blood cell, platelet, lymphocyte, and neutrophil counts of the DOX-iv group were significantly lower than those of the other groups, which was due to the bone marrow suppression caused by intravenous DOX injection. Conversely, the indicators of the HEC-(DOX) group were not significantly different from the blank groups, indicating DOX dropped into the operation site avoided bone marrow suppression. However, the ruptured muscle texture shown in H\&E staining demonstrated that DOX was toxicity to the contacting tissues (Fig S2B). Therefore, it was necessary to develop an effective sustained release formulation of DOX for avoiding it's directly contact to tissue at high concentration.

\section{Reference}

(1) Zhao, L.; Zhou, Y.; Gao, Y.; Ma, S.; Zhang, C.; Li, J.; Wang, D.; Li, X.; Li, C.; Liu, Y.; Li, X. Bovine serum albumin nanoparticles for delivery of tacrolimus to reduce its kidney uptake and functional nephrotoxicity. Int. J. Pharm. 2015, $483 \quad$ (1-2), 180-187. http://doi.org/10.1016/i.ijpharm.2015.02.018. 

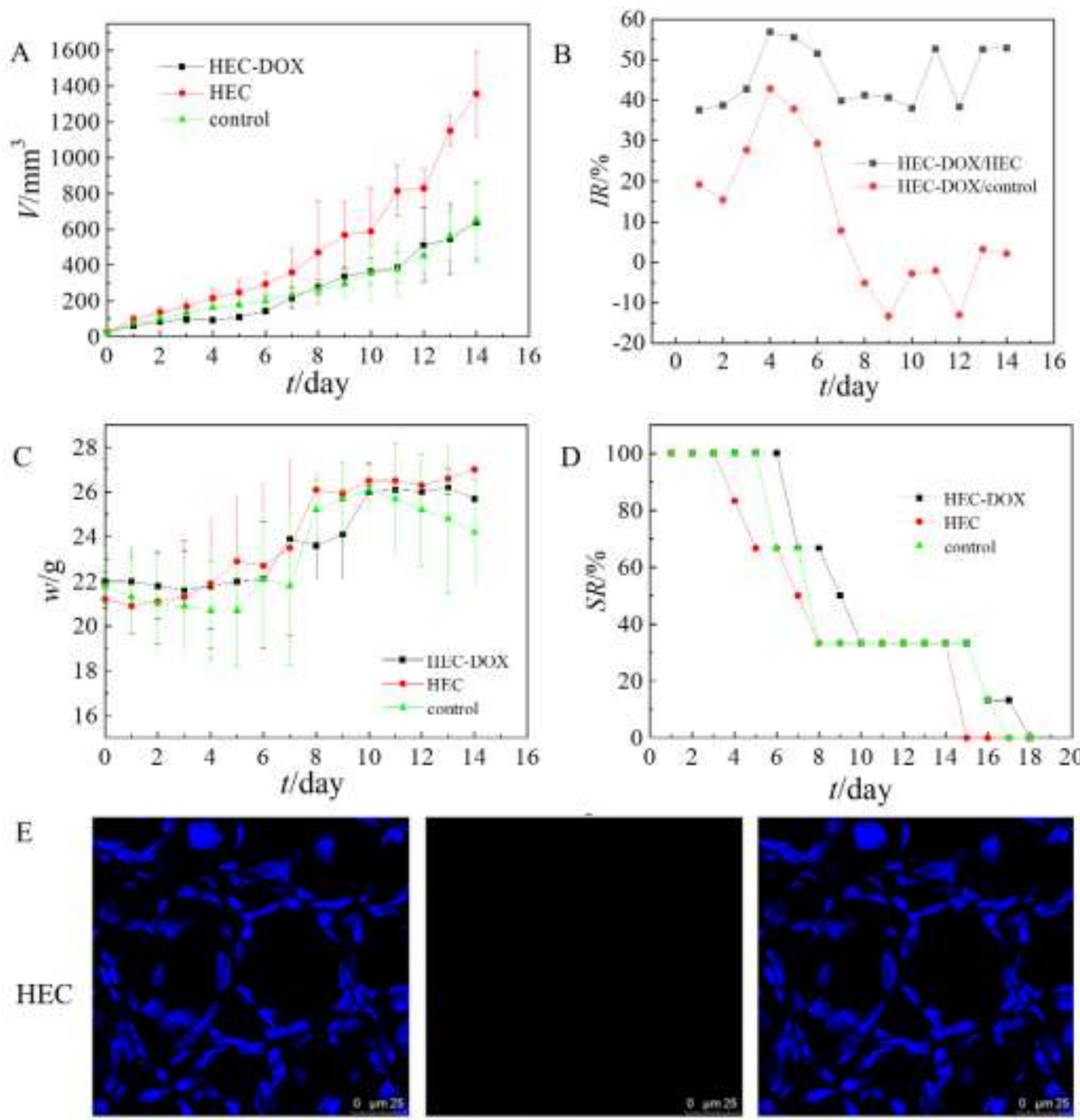

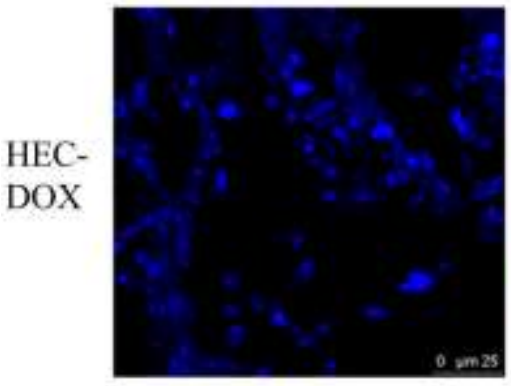

DAPI

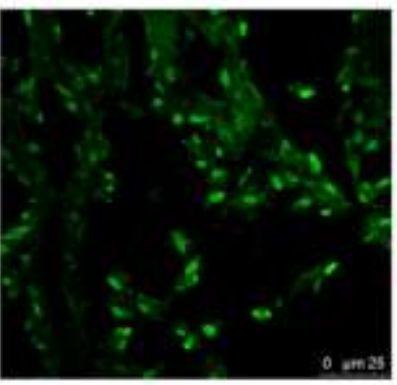

DNA fragments

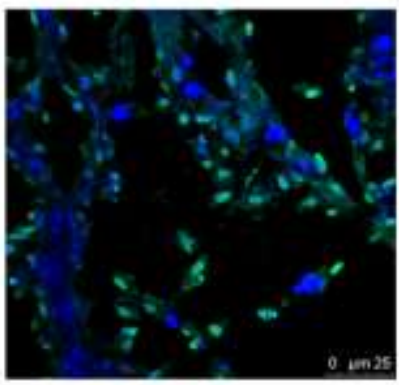

overlay

Fig S1. In vivo antitumor evaluation. (A) Tumor volumes at different time after treatment ( $\mathrm{n}=6)$, (B) Inhibition rate, $(\mathrm{C})$ Body Weight of animals during the treatment, (D) Survival rate of tumor-bearing mice after treatment, (E) TUNEL detection of apoptotic cells in tumor tissues following treatment with different formulations on the 9th day after the first administration. DNA fragments were labeled with fluorescein (green) and nuclei were stained with DAPI (blue). 
A
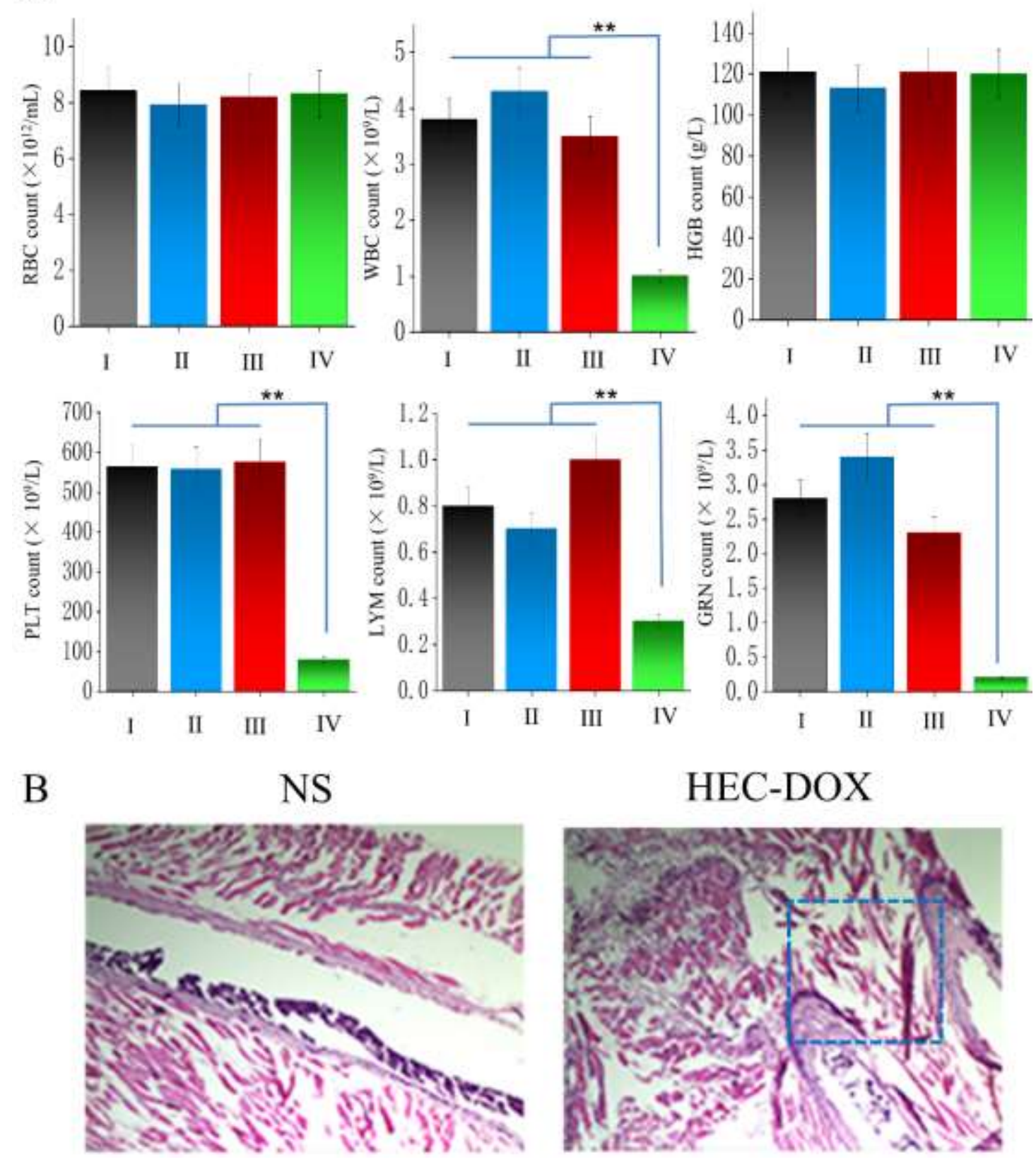

Fig S2. Safety evaluation in vivo. (A) Blood routine test, (B) H\&E staining of organs of interest, bright blue nuclei stained by hematoxylin and pink cytoplasm stained by eosin (Magnification $\times 100$ ).

Note: I. Control, II . HEC, III. HEC-DOX; IV. DOX iv 